\title{
The Book of Chilam Balam of $\mathrm{Na}$, fac-similé, édition et traduction anglaise de Ruth Gubler et David Bolles, Labyrinthos, Lancaster, Calif., 2000, 310 p., gloss.
}

Michel Davoust

\section{OpenEdition}

\section{Journals}

Édition électronique

URL : http://journals.openedition.org/jsa/409

DOI : $10.4000 /$ jsa.409

ISSN : 1957-7842

Éditeur

Société des américanistes

\section{Édition imprimée}

Date de publication : 1 janvier 2001

Pagination : 415-416

ISSN : 0037-9174

Référence électronique

Michel Davoust, "The Book of Chilam Balam of Na, fac-similé, édition et traduction anglaise de Ruth Gubler et David Bolles, Labyrinthos, Lancaster, Calif., 2000, 310 p., gloss. », Journal de la Société des américanistes [En ligne], 87 | 2001, mis en ligne le 17 novembre 2005, consulté le 24 septembre 2020. URL : http://journals.openedition.org/jsa/409 ; DOI : https://doi.org/10.4000/jsa.409

Ce document a été généré automatiquement le 24 septembre 2020.

(c) Société des Américanistes 


\title{
The Book of Chilam Balam of $\mathrm{Na}$, fac- similé, édition et traduction anglaise de Ruth Gubler et David Bolles, Labyrinthos, Lancaster, Calif., 2000, 310 p., gloss.
}

\author{
Michel Davoust
}

1 Les Chilam Balam - livres écrits par des Indiens en langue maya yucatèque mais avec un alphabet latin entre le $\mathrm{XVI}^{\mathrm{e}}$ et le $\mathrm{XIX}^{\mathrm{e}}$ siècle - sont connus sous le nom de la ville dont ils sont originaires. Le Chilam Balam de $\mathrm{Na}$ fait exception à cette règle, puisque $\mathrm{Na}$ est le nom de famille de ses deux auteurs, José María Na et José Secundino Na. Il appartient au groupe d'ouvrages qui, comme le Kaua, l'Ixil et le Tekax ne comportent pas de matières historiques ou mythiques, mais des prédictions astrologiques et des textes médicaux.

2 Ce document comporte les chapitres suivants :

3 - les pronostics de l'année concernant les 7 jours de la semaine associés avec le premier janvier mais non avec les 4 jours porteurs de l'année maya : Oc, Men, Ahau et Chicchan comme dans les Chilam Balam de Kaua, Perez et Tizimin;

4 - les jours des uinal (mois maya de 20 jours) corrélant l'année chrétienne et l'année maya. Le premier de Pop tombant le 16 juillet ;

5 - le compte des jours, avec, pour l'année de 365 jours, le pronostic des jours et le nom des saints ;

6 - les planètes, les signes du zodiaque, leurs influences et des textes concernant les saignées et les purges ;

7 - des textes médicaux ;

8 - des papiers de famille comme les naissances, baptêmes, mariages et décès de membres de la famille Na. 
9 Gubler et Bolles ont ajouté un glossaire des noms de plantes et d'animaux contenus dans le Chilam Balam de Na, utilisant en particulier les ouvrages de Barrera Marín, Barrera Vásquez et López Franco, Nomenclatura etnobotánica maya (1976) et de R. Roys, Ethno-botany of the Maya (1931).

10 Les facsimilés-photographies des pages du manuscrit présentées par Gubler et Bolles sont en général assez lisibles sauf la première et la dernière page.

11 Pour la transcription des textes, les auteurs ont standardisé l'orthographe suivant les usages du XIX ${ }^{\mathrm{e}}$ siècle, par exemple :

12 - le son s est noté par $\mathrm{z}$ au lieu de ç, s et $\mathrm{z}$, dans le manuscrit ;

13 - le son u est noté par u ou w, au lieu de u et v ;

14 - le son i est noté par i au lieu de $\mathrm{i}, \mathrm{j}$, et $\mathrm{y}$.

15 On aurait souhaité que les lignes de chaque texte fussent numérotées comme Edmonson l'avait fait pour les Chilam Balam de Tizimin et de Chumayel et Arzápalo Marín pour le Rituel des Bacab, ce qui aurait permis d'établir une concordance de tous les termes utilisés dans le texte.

16 La traduction, elle, est accompagnée de notes explicatives de bas de page. Dans leur travail de traduction, les auteurs ont utilisé la version espagnole du groupe Dzibil de Mexico pour les textes des Chilam Balam de Chan Cah, de Tekax, mais aussi de Na (1981-1982).

17 Il reste que cette première traduction exhaustive en anglais du Chilam Balam de Na sera utile aux étudiants et à tous les lecteurs des Chilam Balam, permettant une meilleure approche de ces textes, qui reflètent la culture maya de la période coloniale du Yucatán, en particulier l'usage des plantes médicinales et des thérapies traditionnelles.

\section{AUTEURS}

\section{MICHEL DAVOUST}

Centre d'études des langues indigènes d'Amérique, CNRS, Villejuif 